\section{FRI0583 IGG4-RELATED DISEASE AMONG PATIENTS PREVIOUSLY DIAGNOSED WITH IDIOPATHIC RETROPERITONEAL FIBROSIS. A NATIONWIDE DANISH STUDY}

N. Lomborg ${ }^{1}$, M. Jakobsen ${ }^{2}$, C.S. Bode ${ }^{3}$, P. Junker ${ }^{4} .{ }^{1}$ Department of Rheumatology; ${ }^{2}$ Department of Pathology, Vejle Hospital, Vejle; ${ }^{3}$ Department of Pathology; ${ }^{4}$ Department of Rheumatology, Odense University Hospital, Odense, Denmark

Background: IgG4-related disease (lgG4-RD) is a recently recognized systemic disease of unknown etiology and prevalence [1]. Retrospective single center series have provided evidence that $13-63 \%$ of patients with idiopathic retroperitoneal fibrosis (IRPF) could be reclassified as IgG4-RD. Since immunesuppressants or B-cell targeted therapies may halt or reverse progression, early diagnosis and treatment is important to prevent terminal fibrosis.

Objectives: To estimate the occurrence of IgG4 related retroperitoneal fibrosis (RPF) in Danish patients diagnosed with IRPF.

Methods: The National Danish Pathology Register was searched for biopsy codes relating to retroperitoneal tissue and inflammation from 01.01.2004 through 31.12.2013. Patients below 18 years of age, secondary causes, malignancies, infections, and specimens with multinucleated giant cells or granulomas were excluded. Among 724 candidate cases 68 were identified with IRPF. Among these 25 were left out due to small tissue samples, unavailable patient files or lack of consent. Clinical, laboratory data and imagings were reviewed. Paraffinembedded tissue blocks were retrieved from 18 pathology departments. Four sections were prepared and stained with hematoxylin-eosin, Weigerts elastin and IgG4 immunostaining. Histopathologic features suggesting an IgG4-RD background were recorded (table). Cut-off levels for lgG4 positive cells at $>30$ per HPF and IgG4: total IgG ratio at $\geq 40 \%$ were applied. Patients were categorized as IRPF, definite or possible IgG4-RD according to international consensus [2]. Intergroup comparisons were done using Mann-Whitney $U$ test or Chi square test. Results: Forty three patients (29 males), median age 56 years were included among which 19 (44\%) met the criteria for IgG4-RD comprising 7 with definite, 12 with possible IgG4-RD and 24 with IRPF. Biopsies were available from all participants. Extraretroperitoneal manifestations, standard laboratory measures and serum lgG4 (in 5 individuals) did not differ significantly between RPF subsets. Patients with an IgG4: total IgG ratio $\geq 40 \%$ had significantly more histopathologic features of IgG4-RD compared to a ratio $<40 \%$ (table). Patients with $\geq 30$ IgG4 positive cells per HPF had higher numbers of tissue eosinophils than those with lower lgG4+ cell counts.

Table 1. Histopathologic findings in RPF biopsies $(\mathrm{N}=43$ ) according to IgG4: total IgG ratio

\begin{tabular}{lccc}
\hline & $\begin{array}{l}\text { IgG4/Ig ratio } \geq 40 \% \\
\mathrm{~N}=21\end{array}$ & $\begin{array}{c}\text { lgG4/IgG ratio }<40 \% \\
\mathrm{~N}=22\end{array}$ & -value \\
\hline Lymphoplasmacytic infiltrate & 21 & 13 & 0,002 \\
Storiform fibrosis & 12 & 3 & 0,004 \\
Obliterative phlebitis & 5 & 1 & 0,078 \\
Phlebitis without obliteration & 4 & 2 & 0,378 \\
Increased numbers of eosinophils & 13 & 6 & 0,030 \\
\hline
\end{tabular}

Conclusions: A total of $44 \%$ of IRPF patients was diagnosed with IgG4-RD, $16 \%$ with definite and $28 \%$ with possible IgG4-RD. This estimate may be conservative because specimens with multinucleated giant cells or granulomas were excluded. The closer association of IgG4: total IgG ratio $\geq 40$ vs. lower ratios with histopathologic findings supports a direct pathogenic role by IgG4 bearing cells or IgG4 in IgG4 RPF.

References:

[1] Stone JH, Zen Y, Deshpande V. IgG4-related disease. N Engl J Med 2012;366 6: $539-51$

[2] Deshpande V, Zen Y, Chan JK, Yi EE, Sato Y, Yoshino T et al. Consensus statement on the pathology of IgG4-related disease. Mod Pathol 2012;25 9: 1181-92.

Disclosure of Interest: None declared

DOI: 10.1136/annrheumdis-2017-eular.2597

\section{FRI0584 RHEUMATIC AND MUSCULOSKELETAL DISORDERS RELATED TO IMMUNE CHECKPOINT INHIBITORS IN CANCER PATIENTS : A PROSPECTIVE SINGLE-INSTITUTION STUDY}

K. Marie $^{1}$, L. Rouxel ${ }^{1}$, R. Veillon ${ }^{2}$, F. Martin ${ }^{2}$, C. Dutriaux ${ }^{3}$, A. Pham-Ledard ${ }^{3}$, S. Prey $^{3}$, M. Beylot-Barry ${ }^{3}$, M. Gross-Goupil ${ }^{4}$, B. Bannwarth ${ }^{1}$, C. Richez ${ }^{1}$, N. Mehsen-Cetre ${ }^{1}$, T. Schaeverbeke ${ }^{1}$. ${ }^{1}$ Rheumatology, Hôpital Pellegrin; ${ }^{2}$ Pneumology, Hôpital Haut-Lévêque; ${ }^{3}$ Onco-dermatology; ${ }^{4}$ Medical Oncology, Hôpital Saint-André, Bordeaux, France

Background: Immune checkpoint inhibitors (ICI) targeting cytotoxic T-lymphocyteassociated protein 4 (CTLA-4) and programmed cell death protein 1 (PD-1) axis have been a major advance in cancer immunotherapy. By enhancing T cell activity, unprecedented long-lasting tumour responses are observed in selected cancers but some patients experience immune related adverse events (irAE).

Objectives: To evaluate the prevalence and type of rheumatic and musculoskeletal disorders in patients receiving $\mathrm{ICl}$ at a single institution (University Hospital of Bordeaux, France)

Methods: All cancer patients with musculoskeletal disorders while receiving
$\mathrm{ICl}$ were referred to our rheumatology department. For each referred patient, an experienced rheumatologist performed a comprehensive clinical evaluation Blood tests (inflammatory markers, serum creatine kinase, autoantibodies) and imaging (X-rays, ultrasound) were obtained according to clinical findings. HLA-DR phenotyping was also performed for some patients to search for shared epitope. Results: From September 2015 to December 2016, 329 patients received ICl (ant CTLA-4: $n=38$; anti PD-1: $n=251$; anti PD-L1: $n=29$; combination anti CTLA-4/ant PD-1: $n=11$ ) and 21 patients were referred to our rheumatology department $(6.4 \%)$. Mean age was 65 years and cancer types included melanoma $(n=10)$, non small cell lung cancer $(n=9)$, Merkel carcinoma $(n=1)$ and renal carcinoma $(n=1)$. All musculoskeletal disorders occurred in patients receiving anti PD1 (nivolumab: $n=12$; pembrolizumab: $n=6$ ) or anti PD-L1 (avelumab: $n=2$; atezolizumab: $n=1$ ), with a median exposure time of 90 days (range: $1-650$ days). There were two distinct clinical presentations: 1) inflammatory arthritis (IA) mimicking either rheumatoid arthritis $(n=5)$ or polymyalgia rheumatica $(n=8)$ and, 2) non-inflammatory musculoskeletal conditions $(n=8)$. Of note, one patient was anti-CCP positive but negative for RF. Shared epitope HLA-DRB1 *01:01 was present in 4 patients. Eleven patients required corticosteroid therapy with a median dose of $15 \mathrm{mg} /$ day (range: $7-30 \mathrm{mg} /$ day). Non-inflammatory disorders were easily managed with NSAIDs, analgesics and/or physiotherapy. ICI treatment was temporarily discontinued in one patient only, in line with the clinical tria protocol. To date, there was a partial or complete tumour response to $\mathrm{ICl}$ in 10 patients whereas 3 had stable disease and 7 had progressive disease. Tumour response or stable disease was observed in 11 of 12 patients with IA but only in 2 of 8 patients with non-inflammatory conditions.

Conclusions: In our series, patients with immune-related IA mimicking rheumatoid arthritis and polymyalgia rheumatica were responsive to low-to-moderate dose of prednisone and did not require $\mathrm{ICI}$ discontinuation. Furthermore, tumour response was frequently observed in such patients.

Disclosure of Interest: None declared

DOI: 10.1136/annrheumdis-2017-eular.6703

\section{FRI0585 FREQUENT, UNUSUALLY SEVERE, LONG LASTING, LOCAL AND SYSTEMIC PNEUMOCOCCAL VACCINE REACTIONS IN PATIENTS WITH CRYOPYRIN-ASSOCIATED PERIODIC SYNDROMES (CAPS): RESULTS OF A PROSPECTIVE REGISTRY BASED STUDY}

V.K. Jaeger ${ }^{1}$, H.M. Hoffmann ${ }^{2}$, T. van der Poll ${ }^{3}$, H. Tilson ${ }^{4}$, J. Seibert ${ }^{5}$, A. Speziale $^{5}$, G. Junge ${ }^{5}$, K. Franke ${ }^{6}$, E. Vritzali ${ }^{5}$, P.N. Hawkins ${ }^{7}$, J. Kuemmerle-Deschner ${ }^{8}$, U.A. Walker ${ }^{1}$. ${ }^{1}$ University Hospital Basel, Basel, Switzerland; ' University of California at San Diego and Rady Children's Hospital, San Diego, United States; ${ }^{3}$ University of Amsterdam, Amsterdam, Netherlands; ${ }^{4}$ University of North Carolina, Chapel Hill, United States; ${ }^{5}$ Novartis Pharma AG, Basel, Switzerland; ${ }^{6}$ QuintilesIMS, Real World Insights, NC, United States; ${ }^{7}$ University College London, London, United Kingdom; ${ }^{8}$ University Hospital Tübingen, Tübingen, Germany

Background: Pneumococcal, tetanus and influenza vaccinations are recom mended for patients with Cryopyrin-Associated Periodic Syndromes (CAPS) when treated with immunosuppressive medication.

Objectives: This study aims to report the safety of pneumococcal and other vaccinations administered to CAPS patients.

Methods: All CAPS patients followed in the $\beta$-CONFIDENT (Clinical Outcomes and Safety Registry study of llaris ${ }^{\circledR}$ patients) registry between $07 / 2010$ and $12 / 2015$ were analysed if they had received a vaccination. The $\beta$-CONFIDENT registry is a global, long-term, prospective, observational registry, capturing and monitoring CAPS patients treated with canakinumab [1]

Results: 68 CAPS patients (56\% female, age range $3.5-73$ years) had received a total of 159 vaccine injections, 107 injections against influenza, 19 pneumococcal vaccinations (15 pneumococcal polysaccharide vaccines [PPV], 2 pneumococcal conjugate vaccines [PCV], 2 unknown), 12 against tetanus/diphtheria antigens and 21 other vaccinations.

A reaction was observed in 22 vaccine injections (14\%) administered to 18 patients. 13 vaccine reactions (68\% of all pneumococcal vaccine injections) occurred in 12 patients receiving pneumococcal vaccines. 12 PPV injections ( $80 \%$ of all PPV injections) elicited a vaccine reaction while none was elicited by the PCV injections The high frequency of pneumococcal vaccine reactions contrasted with that of the reactions to other vaccine types: only $17 \%$ and $7 \%$ of the tetanus/diphtheria and influenza vaccinations, respectively, elicited a vaccine reaction. The odds ratios to react to the pneumococcal vaccines compared to influenza and tetanus/diphtheria vaccines were 31.0 (95\% confidence interval (CI) 8-119) and 10.8 (95\% Cl 2-74).

Vaccine reactions after pneumococcal vaccinations were more severe and lasted significantly longer compared to the reactions after other vaccinations. Fever was elicited by almost half of all PPV injections. All symptoms after pneumococcal vaccination were observed very rapidly, usually within hours. Unlike the symptoms observed after influenza and tetanus/diphtheria vaccination, which resolved rapidly, the symptoms related to PPV were much more prolonged and in some cases lasted more than 3 weeks. In 2 patients, pneumococcal vaccination also elicited symptoms consistent with systemic inflammation due to CAPS reactivation. All 5 vaccine related serious adverse events were associated with pneumococcal vaccination. 